\title{
Approximation in weighted rearrangement invariant Smirnov spaces
}

\author{
Sadulla Z. Jafarov \\ Department of Mathematics, Faculty of Art and Science, Pamukkale University, 20070 Denizli, Turkey; \\ Institute of Mathematics and Mechanics, Azerbaijan National Academy of Sciences, 9 B. Vaxabzade St., Baku, Az- \\ 1141, Azerbaijan \\ E-mail: sjafarov@pau.edu.tr
}

\begin{abstract}
In the present work, we investigate the approximation problems in weighted rearrangement invariant Smirnov spaces. We prove a direct theorem for polynomial approximation of functions in certain subclasses of weighted rearrangement invariant Smirnov spaces
\end{abstract}

2010 Mathematics Subject Classification. 30E10. 41A10, 41A25, 46E30

Keywords. Conformal mapping, weighted rearrangement Smirnov spaces, Carleson curves, Cauchy singular integral, polynomial approximation, modulus of smoothness.

\section{$1 \quad$ Introduction and main result}

Denote by $\mathcal{M}$ the set of all $\mu$-measurable complex valued functions on $\mathcal{R}$ and let $\mathcal{M}^{+}$be the subset of functions from $\mathcal{M}$ whose values lie in $[0, \infty]$. By $\lambda_{E}$ we denote the characteristic function of a $\mu$-measurable set $E \subset \mathcal{R}$.

Let a function $\rho: \mathcal{M}^{+} \longrightarrow[0, \infty]$ be given. The function $\rho$ is called a function norm if it satisfies the following properties for all functions $f, g, f_{n}(n \in N)$, for all constants $a \geqslant 0$ and for all $\mu$-measurable subsets $E$ of $\mathcal{R}$ :

(1) $\rho(f)=0$ if $f=0 \mu$-a.e. ; $\rho(a f)=a \rho(f), \rho(f+g) \leq \rho(f)+\rho(g)$,

(2) $0 \leq g \leq f \mu-$ a. e., then $\rho(g) \leq \rho(f)$,

(3) $0 \leq f_{n} \uparrow f \mu$-a.e., then $\rho\left(f_{n}\right) \uparrow \rho(f)$,

(4) if $E \subset \mathcal{R}, \mu(E)<\infty$, then $\rho\left(\lambda_{E}\right)<\infty$,

(5) for every set $E \subset \mathcal{R}$ with $\mu(E)<\infty$, then $\int_{E} f d \mu \leq C_{E} \rho(f)$,

where $C_{E}$ is a constant depending on $E$ and $\rho$ but independent of $f$. The collection $X=X(\rho)$ of all functions $f \in \mathcal{M}$ for which $\rho(|f|)<\infty$ is called a Banach function space. For each $f \in X$, the norm of $f$ is defined by

$$
\|f\|_{X}:=\rho(|f|) \text {. }
$$

Note that Banach function space $X$ equipped with the norm $\|f\|_{X}$ is a Banach space [6, pp. 6-7].

If $\rho$ is a function norm, its associate norm $\rho^{\prime}$ is defined on $\mathcal{M}^{+}$by

$$
\rho^{\prime}(f):=\sup \left\{\int_{\mathcal{R}} f g d \mu: f \in \mathcal{M}^{+}, \rho(f) \leq 1\right\}, g \in \mathcal{M}^{+} .
$$

If $\rho$ is a function norm, then $\rho^{\prime}$ is also a function norm [6, pp. 8-9].

Tbilisi Mathematical Journal 9(1) (2016), pp. 9-21.

Tbilisi Centre for Mathematical Sciences.

Received by the editors: 25 February 2014.

Accepted for publication: 15 November 2015 
Let $\rho$ be a function norm and $\rho^{\prime}$ be its associate function norm. The Banach function space $X\left(\rho^{\prime}\right)$ determined by the function norm $\rho^{\prime}$ is called the associate space of $X=X(\rho)$ and is denoted by $X^{\prime}$. Note that every Banach function space coincides with its second associate space $X^{\prime \prime}=\left(X^{\prime}\right)^{\prime}$ and $\|f\|_{X}=\|f\|_{X^{\prime}}$ for all $f \in X \quad[6$, pp. 10-12].

It is well- known that $[6$, p. 9$]$

$$
\|g\|_{X^{\prime}}=\sup \left\{\int_{\mathcal{R}}|f g| d \mu: f \in X,\|f\|_{X} \leq 1\right\} .
$$

Note that for every $f \in X$ and $g \in X^{\prime}$ the following inequality holds [6, Ch.1, Theorem 2.4]:

$$
\int_{\mathcal{R}}|f g| d \mu \leq\|f\|_{X} \quad\|g\|_{X^{\prime}} .
$$

Moreover, it is important fact [6, p.10] that for every $f \in X$

$$
\|f\|_{X}=\sup \left\{\int_{\mathcal{R}}|f g| d \mu: g \in X^{\prime},\|g\|_{X^{\prime}} \leq 1\right\} .
$$

The distribution function $\mu_{f}$ of a measurable function is defined by

$$
\mu_{f}(\lambda):=\text { meas }\{x \in \mathcal{R}:|f(x)|>\lambda\},
$$

for $\lambda \geqslant 0$. Two measurable functions $f$ and $g$ are said to be equimeasurable if $\mu_{f}(\lambda)=\mu_{g}(\lambda)$ for all $\lambda \geqslant 0$.

Definition 1. A function norm $\rho: \mathcal{M}^{+} \longrightarrow[0, \infty]$ is called rearrangement- invariant if for every pair of equimeasurable functions $f, g \in \mathcal{M}^{+}$the equality $\rho(f)=\rho(g)$ holds. In this case, the Banach function space generated by $\rho$ is called a rearrangement invariant space (r.i.space) [6, p. $59]$.

These spaces are sufficiently wide; the Lebesgue, Orlicz, Lorentz spaces are examples of rearrangement invariant spaces. For every rearrangement - invariant space $X[6$, p.78] we have $L_{\infty} \subset X \subset L_{1}$. A Banach function space $X$ is rearrangement- invariant if and only if its associate space $X^{\prime}$ rearrangement-invariant too [6, p. 60].

Note that detailed information on r.i. space can be found in [6], [36], [41] and [42].

Let $f$ be a measurable function. The function $f^{*}$ defined by

$$
f^{*}(t):=\inf \left\{\lambda: \mu_{f}(\lambda) \leq t\right\}, t \geqslant 0
$$

is called the decreasing rearrangement of the function $f$.

Let $X$ be a rearrangement -invariant space. Considering Luxemburg representation theorem [6, pp. 62-64], there is a (not necessarily unique) rearrangement invariant function norm $\bar{\rho}$ over $[0, \infty]$ with the Lebesgue measure $m$ for which

$$
\rho(f)=\bar{\rho}\left(f^{*}\right), f \in \mathcal{M}^{+}
$$


The r.i. space over $[0, \infty]$ with Lebesgue measure $m$ generated by $\bar{\rho}$ is denoted by $\bar{X}$.

For each $x>0$, let us consider the dilation operator $H_{x}$ defined on $\bar{X}$ by

$$
\left(H_{x} f\right)(t):=\left\{\begin{array}{ll}
f(x t), & x t \in[0, \mu(\mathcal{R})] \\
0, & x t \notin[0, \mu(\mathcal{R})]
\end{array}, t>0 .\right.
$$

Let $\mathcal{B}(\bar{X})$ be the Banach algebra of the bounded linear operator on $\bar{X}$. According to [6, pp. 165] $H_{1 / x} \in \mathcal{B}(\bar{X})$. We denote by $h_{X}(x)$ the operator norm of $H_{1 / x}$, i.e.,

$$
h_{X}(x):=\left\|H_{1 / x}\right\|_{\mathcal{B}(\bar{X})} .
$$

Let us consider the limits [36 ], [40]

$$
\alpha_{X}:=\lim _{t \rightarrow o} \frac{\log h_{X}(t)}{\log t}, \quad \beta_{X}:=\lim _{t \rightarrow \infty} \frac{\log h_{X}(t)}{\log t}
$$

By [36] the above limits exist and $\alpha_{X} \leq \beta_{X}$. The numbers $\alpha_{X}$ and $\beta_{X}$ are called the lower and upper Boyd indices of the r.i. space $X$, respectively. Note that for an arbitrary r.i. space the Boyd indices $\alpha_{X}, \beta_{X} \in[0,1]$. The Boyd indices are said to be nontrivial if $0<\alpha_{X} \leq \beta_{X}<1$. Detailed information on properties of the Boyd indices can be found in [6], [8], [42] and [43].

Let $\Gamma$ be a rectifiable Jordan curve in the complex plane $\mathbb{C}$. This curve separates the plane into two domains $G:=\operatorname{int} \Gamma$ and $G^{-}:=\operatorname{ext} \Gamma$. Without loss of generality, we may asume that $0 \in G$. Let $T:=\{w \in \mathbb{C}:|w|=1\}, D:=\operatorname{int} T, D^{-}:=\operatorname{ext} T$ and $w=\varphi(z)$ be the conformal mapping of $G^{-}$onto $D^{-}$normalized by the conditions

$$
\varphi(\infty)=\infty, \lim _{z \rightarrow \infty} \frac{\varphi(z)}{z}>0
$$

and let $\psi:=\varphi^{-1}$ be the inverse mapping of $\varphi$.

We denote by $L_{p}(\Gamma), 1<p<\infty$, the set of all measurable complex valued functions such that $|f|^{p}$ is Lebesgue integrable with respect to the arclength on $\Gamma$. Let $w=\varphi_{1}(z)$ indicate a function that maps the domain $G$ conformally onto the disk $|w|<1$. The inverse mapping of $\varphi_{1}$ will be shown by $\psi_{1}$. Let $\Gamma_{r}$ denote circular images in the domain $G$, that is, curves in $G$ corresponding to circle $\left|\varphi_{1}(z)\right|=r$ under the mapping $z=\psi_{1}(w)$.

We use $c, c_{1}, c_{2}, \ldots$ to denote constants (which may, in general, differ in different relations) depending only on numbers that are not important for the question of our interest.

Definition 2. The analytic function $f$ in domain $G$ will be called a function of the class $E_{p}(G)$ if

$$
\int_{\Gamma_{r}}|f(z)|^{p}|d z|<\infty .
$$

Definition 3. We shall call the $E_{p}(G)$ class the Smirnov class.

It is evident that any analytic function $f$ belonging to the $E_{p}(G)$ class will also belong to the $E_{1}(G)$ class, that is,

$$
\int_{\Gamma_{r}}|f(z)||d z| \leq c<\infty,
$$


informly in $r, 0<r<1$. Since $E_{p}(G) \subset E_{1}(G)$,every function $f \in E_{p}(G)$ has a non-tangential limit almost everywhere (a. e.) on $\Gamma$, and if we use the same notation for the non-tangential limit of $f$, then $f \in L^{p}(\Gamma)$.

$L_{p}(\Gamma)$ and $E_{p}(G)$ are Banach spaces with respect to the norm

$$
\|f\|_{E_{p}(G)}:=\|f\|_{L_{p}(\Gamma)}:=\left(\int_{\Gamma}|f(z)||d z|\right) .
$$

Note that the general information about Smirnov classes can be found in the books [10, pp. 168-185] and [15, pp. 438-453].

A measurable function $\omega: \Gamma \rightarrow[0, \infty]$ is called a weighted function if the preimage $\omega^{-1}\{0, \infty\}$ has measure zero.

Definition 4. The class of measurable functions $f$ defined on $\Gamma$ and satisfying the condition $|f| \omega \in L_{p}(\Gamma), 1<p<\infty$ is called $\omega$-weighted Lebesgue space $L_{p}(\Gamma, \omega)$ with the norm

$$
\|f\|_{L_{p}(\Gamma, \omega)}:=\|f \omega\|_{L_{p}(\Gamma)} .
$$

Definition 5. The $\omega$-weighted Smirnov class $E_{p}(G, \omega)$ is defined as

$$
E_{p}(G, \omega):=\left\{f \in E_{1}(G): f \in L_{p}(\Gamma, \omega)\right\} .
$$

Let $\Gamma$ be a closed rectifiable Jordan curve in the complex plane $\mathbb{C}$. For $t \in \Gamma$ and $r>0$ we denote by $\Gamma(z, \varepsilon)$ the portion of $\Gamma$ in the open disk of radius $\mathrm{r}$ centred at $z$.

Definition 6. [26]. Let $|\Gamma(z, \varepsilon)|$ denote the length (Lebesgue measure) of $\Gamma(z, \varepsilon)$. The curve $\Gamma$ is called a Carleson curve (or regular curve) if

$$
C_{\Gamma}:=\operatorname{supsup}_{z \in \Gamma \varepsilon>0} \frac{1}{\varepsilon}|\Gamma(z, \varepsilon)|<\infty
$$

For instance, convex curves, Ljapunov curves, chord arcs, smooth curves and Lipschitz curves are all regular. We denote by $S$ the set of all Carleson curves in the complex plane.

Definition 7. Let $1<p<\infty, 1<q<\infty$ and $\frac{1}{p}+\frac{1}{q}=1$ and $A_{p}(\Gamma)$ be the collection of all weights on $\Gamma$ satisfying the condition

$$
\operatorname{supsup}_{t \in \Gamma \varepsilon>0}\left(\frac{1}{\varepsilon} \int_{\Gamma(z, r)} \omega^{p}(\tau)|d \tau|\right)^{1 / p}\left(\frac{1}{\varepsilon} \int_{\Gamma(z, r)} \omega^{-q}(\tau)|d \tau|\right)^{1 / q}<\infty
$$

The weight functions which belong to $A_{p}(\Gamma)$ for some $1<p<\infty$, are called the Muckenhoupt weights. 
Let $\Gamma \subset \mathbb{C}$ be a closed rectifiable Jordan curve with the Lebesgue length measure $|d \tau|$ and $X(\Gamma)$ be a r.i. space over $\Gamma$ and $X^{\prime}(\Gamma)$ it is associate space.

Let $\omega$ be a weighted function. We denote by [40] $X(\Gamma, \omega)$ the linear space of all measurable functions $f$ such that $f \omega \in X(\Gamma)$ and set

$$
\|f\|_{X(\Gamma, \omega)}:=\|f \omega\|_{X(\Gamma)} .
$$

Definition 8. A normed space $X(\Gamma, \omega)$ is called a weighted rearrangement - invariant space (w.r.i. space).

Note that according to [6, Section 1.1] and [39] if $\omega \in X(\Gamma, \omega)$ is a Banach function space then its associate space is the Banach function space $X^{\prime}\left(\Gamma, \omega^{-1}\right)$ with the norm $\|f\|_{X^{\prime}\left(\Gamma, \omega^{-1}\right)}=$ $\left\|f \omega^{-1}\right\|_{X^{\prime}(\Gamma)}$. If $\omega \in X(\Gamma)$ and $\omega^{-1} \in X^{\prime}(\Gamma)$, then from Hölder's inequality we obtain

$$
L^{\infty} \subset X(\Gamma, \omega) \subset L^{1}(\Gamma) .
$$

Definition 9. For a weight $\omega$ on $\Gamma$ we denote by $E_{X}(G, \omega)$ the subclass of analytic functions of $E^{1}(G)$ whose boundary value functions belong to the w.r.i space $X(\Gamma, \omega)$.

For $\zeta \in \Gamma$ we define the point $\zeta_{h} \in \Gamma$ by

$$
\zeta_{h}:=\psi\left(\varphi(\zeta) e^{i h}\right), h \in[0,2 \pi] .
$$

Definition 10. Let $\Gamma$ rectifiable Jordan curve, and $f \in X(\Gamma, \omega)$. Then the function $\Omega_{p(.), \omega}^{(2)}(f,$. defined by

$$
\Omega_{\Gamma, X, \omega}^{(2)}(f, \delta):=\delta^{2} \sup _{t \geq \delta} t^{-2} \sup _{|h| \leq t}\left\|f\left(\zeta_{h}\right)+f\left(\zeta_{-h}\right)-2 f(\zeta)\right\|_{X(\Gamma, \omega)}
$$

is called generalized modulus of smoothness in the $X(\Gamma, \omega)$.

We suppose that $\omega(\delta)$ is a nonnegative, continuous, nondecreasing real function satisfying the conditions $\omega(0)=0, \omega(\delta)>0,(\delta>0)$ and $\omega(n \delta) \leq c n \omega(\delta)$, where $n \in \mathbb{N}$ and constant $c>0$. We denote by $H_{\Gamma}^{\omega} E_{X}(G, \omega)$ the class of functions $f \in E_{X}(G, \omega$ for which

$$
\Omega_{\Gamma, X, \omega}^{(2)}(f, \delta)<c \omega(\delta),
$$

where some constant $c$ independent of $f$ and $\delta$.

Using the method of proof in [44] it can be shown easily that if $f, f_{1} \in H_{\Gamma}^{\omega} E_{X}(G, \omega)$, the modulus of smoothness $\Omega_{\Gamma, X, \omega}^{(2)}(f, \delta)$ satisfy the following conditions:

$$
\begin{aligned}
\Omega_{\Gamma, X, \omega}^{(2)}(f, 0) & =0 \\
\Omega_{\Gamma, X, \omega}^{(2)}(f, \delta) & \geq 0 \\
\lim _{\delta \rightarrow 0} \Omega_{\Gamma, X, \omega}^{(2)}(f, \delta) & =0, \\
\Omega_{\Gamma, X, \omega}^{(2)}\left(f+f_{1}, \delta\right) & \leq \Omega_{\Gamma, X, \omega}^{(2)}(f, \delta)+\Omega_{\Gamma, X, \omega}^{(2)}\left(f_{1}, \delta\right) .
\end{aligned}
$$


Under different restrictive conditions upon $\Gamma=\partial G$, the direct problems of approximation theory in non-weighted and weighted Smirnov spaces have been investigated by several authors (see, for example, [48], [1], [2], [38], [21 ], [13], [44], [22], [ 24]-[26] and [29]). The problems of approximation theory for weighted rearrangement invariant spaces are studied in [17], [19], [23] and [51]. In this work, we prove a direct theorem of approximation theory in weighted rearrangement invariant Smirnov spaces. We investigate approximation problems in the class $H_{\Gamma}^{\omega} E_{X}(G, \omega)$. Similar problems of the approximation theory in different spaces have been studied by several authors (see, for example, [3]-[5 ], [9], [16], [18], [30]-[35], [37], [46], [47], [49] and [50]).

Main result in our work is the following theorem.

Theorem 1. Let $\Gamma \in S, \alpha_{X}, \beta_{X}$ be the nontrivial indices and let $\omega \in A_{1 / \alpha_{X}}(\Gamma) \cap A_{1 / \beta_{X}}(\Gamma)$. If $f \in H_{\Gamma}^{\omega} E_{X}(G, \omega)$, then for any $n \in N$ there exists an algebraic polynomial $P(\cdot, f)$ of degree at most $n$ such that

$$
\|f-P(\cdot, f)\|_{X(\Gamma, \omega)} \leq c_{1} \omega(1 / n)
$$

with some constant $c_{1}$ independent of $n$.

Note that Theorem 1 is proved for the first time in the case where $\Gamma$ is a unit circle.

\section{Auxiliary results}

Let $f \in L_{1}(\Gamma)$. Then the functions $f^{+}$and $f^{-}$defined by

$$
f^{+}(z)=\frac{1}{2 \pi i} \int_{\Gamma} \frac{f(\zeta)}{\zeta-z} d \zeta, \quad z \in G
$$

and

$$
f^{-}(z)=\frac{1}{2 \pi i} \int_{\Gamma} \frac{f(\zeta)}{\zeta-z} d \zeta, \quad z \in G^{-}
$$

are analytic in $G$ and $G^{-}$respectively, and $f^{-}(\infty)=0$. Thus the limit

$$
S_{\Gamma}(f)(z):=(P . V .) \frac{1}{2 \pi i} \int_{\Gamma} \frac{f(\zeta)}{\zeta-z} d \zeta:=\lim _{\varepsilon \rightarrow 0} \frac{1}{2 \pi i} \int_{\Gamma \cap\{\zeta:|\zeta-z|>\varepsilon\}} \frac{f(\zeta)}{\zeta-z} d \zeta
$$

exists and is finite for almost all $z \in L$.

The quantity $S_{\Gamma}(f)(z)$ is called the Cauchy singular integral of $f$ at $z \in \Gamma$. The linear operator $S_{\Gamma}: f \rightarrow S_{\Gamma} f$ is called the Cauchy singular operator.

According to the Privalov's theorem [15, p. 431] if one of the functions $f^{+}$or $f^{-}$has the non-tangential limits a. e. on $\Gamma$, then $S_{\Gamma}(f)(z)$ exists a. e. on $\Gamma$ and also the other one has non-tangential limits a. e. on $\Gamma$. Conversely, if $S_{\Gamma}(f)(z)$ exists a. e. on $\Gamma$, then the functions $f^{+}$ and $f^{-}$have non-tangential limits a. e. on $\Gamma$. In both cases, the formulae

$$
f^{+}(z)=S_{\Gamma}(f)(z)+\frac{1}{2} f(z), \quad f^{-}(z)=S_{\Gamma}(f)(z)-\frac{1}{2} f(z)
$$


and hence

$$
f=f^{+}-f^{-}
$$

holds a. e. on $\Gamma$.

Note that the class of all regular curves is very wide. G. David proved [12] that $\Gamma$ is a regular curve if and only if for every $f \in L_{p}(\Gamma), S_{\Gamma}(f)$ exists and belongs to $L_{p}(\Gamma)$ and the singular operator $S_{\Gamma}(f): L_{p}(\Gamma) \rightarrow L_{p}(\Gamma)$ is bounded, that is, there exists a constants $c_{2}(p, \Gamma)$ such that

$$
\left\|S_{\Gamma}(f)\right\|_{L_{p}(\Gamma)} \leq c_{2}(p, \Gamma)\|f\|_{L_{p}(\Gamma)}
$$

for all $f \in L_{p}(\Gamma)$. In [20], V.Havin proved that if the singular operator $S_{\Gamma}(f): L_{p}(\Gamma) \rightarrow L_{p}(\Gamma)$ is bounded, for every $f \in L_{p}(\Gamma)$, the functions $f^{+}$and $f^{-}$defined by the formulae (2) and (3) belong to Smirnov's classes $E_{p}(G)$ and $E_{p}\left(G^{-}\right)$, respectively.

We need the following results.

Lemma 1 [45, p. 208]. In order to represent $f$ according to its boundary values in the form Cauchy integral, it is necessary and sufficient that $f \in E_{1}(G)$.

The following theorem, given in [7, pp. 117-144] and [14, p. 89] characterizes the weight functions for which $S_{\Gamma}$ is bounded in the weighted Lebesgue spaces $L_{p}(\Gamma, \omega)$.

Theeorem 2. Let $\Gamma$ be a Carleson curve, $1<p<\infty$, and let $\omega$ be a weight function on $\Gamma$. The inequality

$$
\left\|S_{\Gamma}(f)\right\|_{L_{p}(\Gamma, \omega)} \leq c_{3}(p, \Gamma)\|f\|_{L_{p}(\Gamma, \omega)}
$$

holds for every $f \in L_{p}(\Gamma, \omega)$ if and only if $\omega \in A_{p}(\Gamma)$.

The following theorem associated with boundedness of the Cauchy singular integral operator $S_{\Gamma}$ in weighted rearrangement -invariant spaces holds [40]:

Theorem 3. Let $X(\Gamma)$ be an r.i. space with nontrivial Boyd indices $\alpha_{X}, \beta_{X}$. If a weight $\omega$ belongs to the Muckenhoupt classes $A_{\frac{1}{\alpha_{X}},}(\Gamma)$ and $A_{\frac{1}{\beta_{X}},}(\Gamma)$, then the operator $S_{\Gamma}$ is bounded in the w.r.i space $X(\Gamma, \omega)$.

\subsection{Proof of the main result}

Proof of Theorem 1. We set

$$
F\left(z_{h}\right)=f\left(z_{h}\right)+f\left(z_{-h}\right) .
$$

From the condition of Theorem 1 we have $f(z) \in X(\Gamma, \omega)$ and $S_{\Gamma}$ is bounded in the space $X(\Gamma, \omega)$ and $\left\|f\left(z_{h}\right)+f\left(z_{-h}\right)-2 f(z)\right\|_{X(\Gamma, \omega)}<\infty$. In this case according to [39] and [40] $\omega \in X(\Gamma)$ and 
$1 / \omega \in X^{\prime}(\Gamma)$. Then, from the Hölder inequality for the $X(\Gamma)$ spaces we have

$$
\begin{aligned}
\int_{\Gamma}\left|F\left(z_{h}\right)\right||d z| & =\int_{\Gamma} \frac{\left|f\left(z_{h}\right)+f\left(z_{-h}\right)-2 f(z)+2 f(z)\right|}{|\omega|}|\omega||d z| \\
& \leq \int_{\Gamma} \frac{\left|f\left(z_{h}\right)+f\left(z_{-h}\right)-2 f(z)\right|}{|\omega|}|\omega||d z| \\
+2 \int_{\Gamma} \frac{|f(z)|}{|\omega|}|\omega||d z| & \leq\left\|f\left(z_{h}\right)+f\left(z_{-h}\right)-2 f(z)\right\|_{X(\Gamma, \omega)}\left(\left\|\frac{1}{\omega}\right\|_{X^{\prime}(\Gamma)}\right) \\
+2\|f\|_{X(\Gamma, \omega)}\left(\left\|\frac{1}{\omega}\right\|_{X^{\prime}(\Gamma)}\right) & <\infty
\end{aligned}
$$

It follows from the last inequality, that $F\left(z_{h}\right) \in L_{1}(\Gamma)$. Since $F\left(z_{h}\right) \in L_{1}(\Gamma)$ and $S_{\Gamma}$ is bounded in the space $X(\Gamma, \omega)$, singular integral $S_{\Gamma}\left(F_{h}\right)(z)$ in principal value meaning exists a. e. on $\Gamma$. Then the function is approximed with Jackson-Dzydyk polynomial [11, p.440]. We represent the polynomial Jackson-Dzydyk in the form

$$
P_{n}(z)=\frac{1}{2 \pi} \int_{0}^{\pi} K_{n}(h)\left[S_{\Gamma}\left(F_{h}\right)(z)\right] d h+\frac{1}{4 \pi} \int_{0}^{\pi} K_{n}(h)\left[f\left(z_{h}\right)+f\left(z_{-h}\right)\right] d h,
$$

where $K_{n}(h)$ is a kernel, which is trigonometric polynomials of degree not exceeding $n$ and satisfies the conditions [11, p. 428]

$$
\begin{gathered}
\frac{1}{2 \pi} \int_{-\pi}^{\pi} K_{n}(t) d t=1, \quad(n=0,1,2, \ldots), \\
\int_{-\pi}^{\pi}\left|K_{n}(t)\right| d t \leq c_{4}, \quad(n=0,1,2, \ldots), \\
\int_{-\pi}^{\pi}|t|^{k}\left|K_{n}(t)\right| d t \leq c_{5}(k)(n+1)^{-k} \\
\int_{-\pi}^{\pi}\left(|t|+\frac{1}{n}\right)^{k}\left|K_{n}(t)\right| d t \leq c_{6}(k) n^{-k},(n=1,2, \ldots) .
\end{gathered}
$$

Note that Jackson kernel satisfies the conditions (5)- (8). If $f \in E_{X}(G, \omega)$, it follows that $f \in E_{1}(G)$. Then according to Lemma 1 , the function $f \in E_{1}(G)$ can be written as Cauchy integral

$$
f(z)=\frac{1}{2 \pi i} \int_{\Gamma} \frac{f(\zeta)}{\zeta-z} d \zeta, z \in G
$$


Since $S_{\Gamma}$ is bounded in the space $X(\Gamma, \omega)$, the $S_{\Gamma}(f)(z)$ singular integral exists. Then, for the function $f$ the following identity

$$
f(z)=\left(S_{\Gamma} f\right)(z)+f(z) / 2
$$

holds a. e. on $\Gamma$. Taking into account the last relation, we have

$$
f(z)=\frac{1}{2 \pi} \int_{0}^{\pi} K_{n}(h) f(z) d h+\frac{1}{\pi} \int_{0}^{\pi} K_{n}(h)\left[\left(S_{\Gamma} f\right)(z)\right] d h .
$$

Consideration of (4) and (9) gives us

$$
\begin{aligned}
\left\|f-P_{n}\right\|_{X(\Gamma, \omega)} & \leq \| \frac{1}{2 \pi} \int_{0}^{\pi} K_{n}(h) f(z) d h+\frac{1}{\pi} \int_{0}^{\pi} K_{n}(h)\left[\left(S_{\Gamma} f\right)(z)\right] d h P_{n}(z) \\
& -\frac{1}{2 \pi} \int_{0}^{\pi} K_{n}(h)\left[S_{\Gamma}\left(F_{h}\right)(z)\right] d h- \\
& \frac{1}{4 \pi} \int_{0}^{\pi} K_{n}(h)\left[f\left(z_{h}\right)+f\left(z_{-h}\right)\right] d h \|_{X(\Gamma, \omega)} .
\end{aligned}
$$

Using (10), Minkowski's inequality and the boundedness of singular operator $S_{\Gamma}$ we get

$$
\begin{aligned}
\left\|f-P_{n}\right\|_{X(\Gamma, \omega)} & \leq c_{7}\left\|\int_{0}^{\pi} K_{n}(h)\left[S_{\Gamma}\left(F_{h}-2 f\right)(z)\right] d h\right\|_{X(\Gamma, \omega)} \\
+c_{8}\left\|\int_{0}^{\pi} K_{n}(h)\left[\left(F_{h}-2 f\right)(z)\right] d h\right\|_{X(\Gamma, \omega)} & \leq c_{9} \int_{0}^{\pi} K_{n}(h) d h\left\|S_{\Gamma}\left(F_{h}-2 f\right)(z)\right\|_{X(\Gamma, \omega)} \\
+c_{10} \int_{0}^{\pi} K_{n}(h) d h\left\|\left(F_{h}-2 f\right)(z)\right\|_{X(\Gamma, \omega)} & \leq c_{11} \int_{0}^{\pi} K_{n}(h)\left\|\left(F_{h}-2 f\right)(z)\right\| d h_{X(\Gamma, \omega)} \\
+c_{12} \int_{0}^{\pi} K_{n}(h) d h\left\|\left(F_{h}-2 f\right)(z)\right\|_{X(\Gamma, \omega)} & \leq c_{13} \int_{0}^{\pi} K_{n}(h) \Omega_{\Gamma, X, \omega}^{(2)}(h, f) d h \\
& \leq c_{14} \Omega_{\Gamma, X, \omega}^{(2)}(h, f) \int_{0}^{\pi} K_{n}(h)(n h+1) d h . \leq c_{15} \omega(1 / n) .
\end{aligned}
$$

According to (5), (7) and (11), we obtain the inequality (1) of Theorem 1.

Acknowledgement. The author would like to thank referee for all precious advices and very helpful remarks. 


\section{References}

[1] S. Y. Alper, Approximation in the mean of analytic functions of class $E^{p}$, In: Investigations on the Modern Problems of the Function of a Complex Variable, Gos. Izdat. Fiz.-Mat. Lit., Moscow, 1960, pp. 272-286 (in Russian).

[2] J. - E. Andersson, On the degree of polynomial approximation in $E^{p}(D)$, J. Approx.Theory 19 (1977), 61-68.

[3] V. V. Andrievskii, Approximation of functions by partial sums in Faber polynomials on continua with a nonzero local geometric characteristic, Ukrain. Mat. Zh. 32 (1980), no. 1, 3-10 (in Russian

[4] V. V. Andrievskii, Weighted uniform polynomial approximation and moduli of smoothness on continuain complex plane, J. Approx. Theory 164 (2012), 297-319..

[5] R. Akgün, D. M. Israfilov, Approximation and moduli of fractional orders in Smirnov-Orlicz classes, Glas. Mat. 43 (63) (2008), 121-136.

[6] C. Bennett and R. Sharpley, Interpolation of Operators, Pure and Applied Mathematics, 129, Academic Press. Inc., Boston, MA, 1988.

[7] A. Böttcher and Yu. I. Karlovich, Carleson Curves, Muckenhoupt Weights and Teoplitz Operators, Progress in Mathematics, 154, Birkhäuser Verlag, Basel, 1997.

[8] D. W. Boyd, Indices of function space and their relationship to interpolation, Canad. J. Math. 21 (1969), 1245-1254.

[9] A. Çavus and D. M. Israfilov, Approximation by Faber-Laurent rational functions in the mean of functions of the class $L_{p}(\Gamma)$ with $1<p<\infty$, Approximation Theory App. 11 (1995), 105-118.

[10] P. L. Duren, Theory of $H^{p}$ Spaces, Academic Press, 1970.

[11] V. K. Dzyadyk, Introduction to the Theory of Uniform Approximation of Functions by Means of Polynomials, Nauka, Moscow, 1977 (in Russian).

[12] G. David, Operateurs integraux singulers sur certaines courbes du plan complexe, Ann. Sci. Ecol. Norm. Super. 4 (1984), 157-189.

[13] E. M. Dyn'kin, The rate of polynomial approximation in complex domain, pp. 90-142. In: Complex Analysis and Spectral Theory (Lenilngrad, 1979/1980), Springer-Verlag, Berlin.

[14] E. M. Dyn'kin and B. P. Osilenker, Weighted norm estimates for singular integrals and their applications, Itogi Nauki Tekh., Ser. Mat. Anal. 21 (1983), 42-129 (in Russian)

[15] G. M. Golusin, Geometric Theory of Functions of a Complex Variable, Traslation of Mathematical Monographs, 26, Providence, RI: AMS, 1968.

[16] A. Guven, D. M. Israfilov, Polynomial approximation in Smirnov- Orlicz classes, Computational Methods and Function Theory, 2 (2002), no. 2, 509-517. 
[17] A. Guven, D.M. Israfilov, Approximation in rearrangement invariant spaces in Carleson curves, East Journal on Approximations, 12 (4) (2006), 381-395.

[18] A. Guven, D. M. Israfilov, Rational approximation in Orlicz spaces on Carleson curves, Bull. Belg. Math. Soc. 12 (2005), 223-234.

[19] A. Guven, D. M. Israfilov, Approximation by trigonometric polynomials in weighted rearrangement invariant spaces, Glas. Mat. Ser. 44 (64) (2009), 423-446.

[20] V. P. Havin, Continuity in $L_{p}$ of an integral operator with the Cauchy kernel (Russian, English Summary).Vestnik Leningrad Univ. 22 (1967), no 7, 103 MR 36 \#, 1623.

[21] I. I. Ibragimov and D. I. Mamedkhanov, A constructive characterization of a certain class of functions, Dokl. Akad. Nauk SSSR 223 (1975), no. 35-37; Soviet Math. Dokl. 4 (1976), $820-823$.

[22] D. M. Israfilov, Approximate properties of the generalized Faber series in an integral metric, Izv. Akad. Nauk Az. SSR, Ser. Fiz.-Tekh. Mat. Nauk 2 (1987), 10-14 (in Russian).

[23] D. M. Israfilov, R. Akgün, Approximation by polynomials and rational functions in weighted rearrangement invariant spaces, J. Math. Anal. Appl. 346 (2008) 489-500.

[24] D. M. Israfilov, Approximation by p-Faber polynomials in the weighted Smirnov class $E^{p}(G, \omega)$ and the Bieberbach polynomials, Constr.Approx. 17 (2001), no. 3, 335-351.

[25] D. M. Israfilov, Approximation by p-Faber-Laurent rational functions in the weighted Lebesgue spaces, Czechoslovak Math. J. 54 (129) (2004), no. 3, 751-765.

[26] D. M. Israfilov, A. Guven, Approxmation in weighted Smirnov classes, East J. Approx. 11 (2005), no.1 91-102.

[27] D. M. Israfilov, B. Oktay, R. Akgün, Approximation in Smirnov-Orlicz classes, Glas. Mat. Ser. 40 (60) (2005), no. 1, 87-102.

[28] D. M. Israfilov, V. Kokilashvili, S. Samko, Approximation in weighted Lebesgue and Smirnov spaces with varible exponents, Proc. A. Razmadze Math. Inst. 143 (2007), 25-35.

[29] D. M. Israfilov, Approximate properties of the generalized Faber series in an integral metric,Izv. Akad. Nauk Azerb. SSR, Ser. Fiz.-Tekh. Math. Nauk 2 (1987), 10-14 (in Russian).

[30] S. Z. Jafarov, A direct and an inverse theorem in the theory of approximation on quasiconformal curves, Izv. Akad. Nauk Azerb. SSR Ser. Fiz-Tekhn. Mat. Nauk 8 (1987), no. 4, 8-11 (in Russian).

[31] S. Z. Jafarov, Approximation of functions by rational functions on closed curves of the complex plane, Arab. J. Sci.Eng. 36 (2011), no.8, 1529-1534.

[32] S. Z. Jafarov, Approximation by polynomials and rational functions in Orlicz spaces, J. Comput. Anal. Appl. (JoCAAA) 13 (2011), no.5, 953-962 
[33] S. Z. Jafarov, Approximation by rational functions in Smirnov-Orlicz classes, J. Math. Anal. Appl. 379 (2011), no. 2, 870-877.

[34] S. Z. Jafarov, On approximation in weighted Smirnov-Orlicz classes, Complex Var. Elliptic Equ. 57 (2012), no. 5, 567-577.

[35] S. Z. Jafarov, The inverse theorem of approximation of the functions in Smirnov-Orlicz classes, Math. Inequal. Appl 12 (2012) no. 4, 835-844.

[36] S. G. Krein, Ju. I. Petunin, and E. M. Semenov, Interpolation of linear operators, Nauka, Moscow, 1978 (Russian), English translation: Ams Translation of Mathematical Monographs 54, Providenie, R. I., 1982.

[37] V. M. Kokilashvi, On analytic functions of Smirnov- Orlicz classes, Studia Math. 31 (1968) 43-59.

[38] V. M. Kokilashvili, A direct theorem on mean approximation of analytic functions by polynomials, Soviet Math. Dokl. 10 (1969), 411-414.

[39] A. Yu. Karlovich, Algebras of Singular integral operators on rearrangement invariant spaces and Nikolski ideals, New York J. Math. 8 (2002), 215-234.

[40] A.Yu. Karlovich, Algebras of singular integral operators with PC coefficients in rearrangementinvariant spaces with Muckenhoupt weights, J. Operator Theory 47 (2002), 303-323.

[41] A.Yu. Karlovich, Singular integral operators with piecewise continuous coefficients in reflexive rearrangement invariant spaces, Integral Equations Operator Theory 32 (1998), 436-481.

[42] J. Lindenstrauss and L. Tzafriri, Classical Banach Spaces. II: Function Spaces, Springer Verlag, New York, Berlin, 1979.

[43] L. Maligranda, Indices and Interpolation, Dissertationes Math. (Rozprawy Mat.), 234 (1985), $1-49$.

[44] D. I. Mamedkhanov, Approximation in complex plane and singular operators with a Cauchy kernel, Dissertation Doct. Phys-math.nauk The University of Tbilisi, 1984 (in Russian).

[45] I. I. Privalov, Boundary Properties of Analytic Functions, Moscow University Press, Moscow, Russia, 1950 (in Russian).

[46] A.-R. K. Ramazanov, On approximation by polynomials and rational functions in Orlicz spaces, Analysis Mathematica, 10 (1984), 117-132.

[47] I. I. Sharapudinov, Approximation of functions in the metric of the space $L^{p(t)}([a, b])$ and quadrature (in Russian), Constructiver function theory 81 (Varna, 1981), 189-193, Publ. House Bulgar. Acad. Sci., Sofia, 1983.

[48] J. L. Walsh and H. G. Russel, Integrated continuity conditions and degree of approximation by polynomials or by bounded analytic functions, Trans. Amer.Math. Soc. 92, (1959), 355-370. 
[49] Y. E. Yildirir, D. M. Israfilov, Simultaneous and converse approximation theorems in weighted Lebesgue spaces, Math. Inequal. Appl. 14 (2011), no. 2, 359-371.

[50] Y. E. Yildirir, R. Cetintas, Approximation theorems in weighted Orlicz spaces, J. Math. Sci. Adv. Appl. 14 (2012), no. 1, 35-49.

[51] H. Yurt, A. Guven, On rational approximation of functions in rearrangement invariant spaces, Journal of Classical Analysis, 3 (2013), no. 1, 69-83. 